\title{
WIDERSPRUCH UND AUFHEBUNG IN DER ENTWICKLUNG DER PHYSIK
}

\section{Martin Strauss (Berlin)}

Nach bürgerlicher Geschichtsschreibung hat es den Anschein, als ob die Entwicklung der Physik vor allem durch die genialen Einfälle einzelner großer Physiker bestimmt sei; dieser Eindruck wird durch verschiedene ,,wissenschaftliche Selbstbiographien“ noch verstärkt. Demgegenüber wird hier der Standpunkt vertreten, daß auch die Entwicklung der Physik objektiven Gesetzen unterliegt und somit im Prinzip bis zu einem gewissen Grade voraussehbar ist, natürlich nicht in den Einzelheiten, aber der Entwicklungstendenz nach. Die Kenntnis der Entwicklungstendenzen oder Entwicklungsgesetze erleichtert nicht nur die Orientierung in den aktuellen Streitfragen der Physik, sondern kann auch ein wichtiges Hilfsmittel in der Planung der Forschung werden.

Das Studium der Geschichte der Physik zeigt, daß die allgemeinen Entwicklungsgesetze auch für die Entwicklung der Physik gelten. Das grundlegende Entwicklungsgesetz ist das der Entfaltung und Uberwindung der Widersprüche. In der Physik treten folgende Arten von Widersprüchen auf:

1. Widersprüche zwischen Theorie und beobachteten Tatsachen (,äußere Widersprüche“);

2a. Widersprüche zwischen verschiedenen Teilen der jeweils akzeptierten Theorie oder, wie wir gewöhnlich sagen: zwischen verschiedenen gleichzeitig akzeptierten Theorien (z. B. zwischen Thermodynamik und Maxwellscher Elektrodynamik: ,Ultraviolett-Katastrophe" beim Problem der spektralen Energieverteilung der Wärmestrahlung); 
2b. Widersprüche, die sich bei der Anwendung einer Theorie auf bestimmte Probleme ergeben (z. B. ,kosmologisches Paradoxon" der Newtonschen Theorie, Gibbssches Paradoxon der klassischen statistischen Thermodynamik, ,explodierendes Elektron“, Kleinsches Paradoxon in der ersten Fassung der Diracschen Theorie des Elektrons, Divergenzen der Quantenfeldtheorie).

Die Widersprüche von der Art $2 \mathrm{a}$ und $2 \mathrm{~b}$ können zusammen als ,innere Widersprüche" bezeichnet werden.

Während die Bedeutung der ,äußeren Widersprüche" für die Entwicklung der Theorie allgemein anerkannt ist, gelten die ,innern Widersprüche" oft als Schönheitsfehler, die man durch geringfügige Abänderungen der Theorie beseitigen müsse. Die Geschichte lehrt jedoch, daß die meisten dieser inneren Widersprüche erst durch eine völlig neuartige Theorie überwunden werden; sie sind daher als Entwicklungskeime, als Fingerzeige für die weitere Entwicklung der theoretischen Grundlagen zu betrachten. Etwas Ähnliches gilt übrigens auch für die äußeren Widersprüche.

Bevor die neue Theorie gefunden ist, kommt es gewöhnlich zu Zwischenlösungen mit Hilfe von ad-hoc-Hypothesen. Typische Beispiele sind die Lorentzsche Kontraktionshypothese, die BohrSommerfeldschen Quantenbedingungen, und die Renormierung in der Quantentheorie der Felder. Derartige Methoden, mit den (inneren oder äußeren) Widersprüchen schon im Rahmen der alten Theorie fertig zu werden bzw. sie ,unschädlich“" zu machen, können als partielle Antizipation der neuen Theorie bewertet werden. Auch die Einführung der sog. Bose-Einstein- bzw. der Fermi-Dirac-Statistik in die Quantenmechanik ist eine partielle Antizipation einer tiefer liegenden Theorie, nämlich der Quantentheorie der Felder.

Die neue Theorie, die als Lōsung der alten Widersprüche entsteht, stellt stets eine Verallgemeinerung oder besser: Aufhebung der alten Theorie dar. Die alte Theorie wird dabei prinzipiell außer Kraft gesetzt und durch die neue ersetzt. Zugleich werden jedoch einige wesentliche Züge der alten Theorie in die neue übernommen. Der Ausdruck ,Verallgemeinerung" bezieht sich auf den mathematischen Apparat und das Axiomensystem der neuen Theorie im Vergleich zur alten; er drückt auch aus, daß der Gültigkeitsbereich der neuen Theorie größer ist als der der alten. Er bringt jedoch ungenügend zum Ausdruck, daß 
vom Standpunkt der neuen Theorie die alte Theorie begrifflich entartet ist, d. h. daß in ihr verschiedene Begriffe dem Umfang nach zusammenfallen (z. B. ,System- oder Koordinatenzeit“ und „Eigenzeit", oder ,Wellenfunktion“ und „Zustandsfunktion“, die in der Quantenmechanik zusammenfallen, nicht aber in der Quantentheorie der Felder). Die neue Theorie ist gerade auch insofern eine Aufhebung der alten Theorie als sie begriffliche Entartungen aufhebt (beseitigt).

Das allgemeine Entwicklungsgesetz - Entfaltung und Uberwindung der Widersprüche - fällt in der Geschichte der Physik weitgehend mit der Tendenz zur Vereinheitlichung der Grundlagen zusammen. So hat z. B. die Spezielle Relativitätstheorie den Widerspruch zwischen Mechanik und Elektrodynamik beseitigt und darüber hinaus ein gemeinsames kinematisches Fundament für alle Teile der Physik geschaffen, während die Quantentheorie u. a. die Widersprüche zwischen Thermodynamik und der übrigen Physik, insb. der Elektrodynamik (,Ultraviolettkatastrophe“) beseitigt hat. Tatsächlich entstand die Quantentheorie aus dem Bemühen Plancks, die Thermodynamik mit der Theorie des elektromagnetischen Feldes zu vereinen. ${ }^{1}$ Das subjektive Streben der Forscher nach einem einheitlichen physikalischen Weltbild ist somit auch objektiv gerechtfertigt; es erweist sich jedoch nur in dem Maße als wirklich progressiv und erfolgreich wie es mit der objektiven Tendenz d.h. mit der jeweiligen geschichtlichen Notwendigkeit und Möglichkeit, übereinstimmt. Gelegentlich schießt jedoch das subjektive Streben nach Vereinheitlichung der Grundlagen über das zur gegebenen Zeit Mögliche hinaus; es entstehen dann gewöhnlich spekulative Fehlgeburten wie etwa die Phlogiston-Theorie, die mechanische Theorie des "Lichtäthers" oder die verschiedenen ,einheitlichen Feldtheorien“. Mitunter schlägt dabei das berechtigte Streben nach Vereinheitlichung in den metaphysischen Versuch um, die ,Weltformel“ - man kann auch sagen: die Formel, nach der Gott die Welt geschaffen hat - zu erraten. Derartige Tendenzen, die für den objektiven Idealismus charakteristisch sind, finden sich bekanntlich z. B. bei Eddington und in dem Spätwerk Einsteins; sie sind auch der eigentliche Grund für Einsteins ablehnende Haltung gegenüber der Quantenmechanik gewesen.

${ }^{1}$ Fũr eine ausführliche Darstellung vgl.: Planck und die Entstehung der Quantentheorie, Jubiläumsfestschrift der Humboldt-Universität, 1960. 
Den meisten der heute lebenden Physikern erscheint die Naturerkenntnis als ein ProzeB, dessen Ende nicht abzusehen ist und in dem Perioden der revolutionären Umgestaltung mit solchen der Konsolidierung der neuen Theorie abwechseln. Die Triebkräfte für die Umgestaltung sind gewöhnlich die äußeren Widersprüche - eine Ausnahme bildet hier die Einsteinsche Gravitationstheorie. Für das Auffinden der neuen Theorie gewinnen jedoch die inneren Widersprüche in dem Maße an heuristischer Bedeutung, in dem die begrifflichen Grundlagen der Theorie sich immer mehr von denen der „Physik des Alltags" entfernen. Damit wächst nämlich nicht nur die Bedeutung der wissenschaftlichen Abstraktion im Vergleich zur Induktion (was allgemein anerkannt ist), sondern auch die heuristische Bedeutung der schon besteheuden Theorie im Vergleich zu der der neuentdeckten Tatsachen. Anders ausgedrückt: der Theoretiker hat heute bei der Aufstellung einer neuen Theorie von vornherein zwei Komplexe zu berücksichtigen: die neuen experimentellen Tatsachen und die schon bestehende Theorie mit ihren inneren Widersprüchen, und seine Aufgabe besteht darin, eine solche Verallgemeinerung oder „Aufhebung“ der bestehenden Theorie zu finden, welche den neuen experimentellen Tatsachen Rechnung trägt. Da nun die neuen experimentellen Tatsachen die Richtung der Verallgemeinerung gewöhnlich nicht eindeutig erkennen lassen, und andererseits die inneren Widersprüche selbst ein Ausdruck des provisorischen Charakters der jeweils erreichten Erkenntnisstufe sind, fällt die Aufgabe der Uberwindung der inneren Widersprüche gewöhnlich weitgehend mit der der theoretischen Verarbeitung des neuen experimentellen Materials zusammen. Tatsächlich war dies schon bei der Quantentheorie und der Speziellen Relativitätstheorie so, nur erkannte man damals erst nachträglich, daß es sich in beiden Fällen um die Beseitigung innerer Widersprüche gehandelt hat.

Heute steht vor den Theoretikern die Aufgabe, die bestehende Quantentheorie der Felder durch eine Theorie der Elementarteilchen aufzuheben. Das Besondere der Situation scheint mir im folgenden zu liegen. Einmal fehlen die äußeren Widersprüche fast vollständig, während die inneren Widersprüche einigermaßen gut bekannt und erforscht sind. Zum anderen erwartet man von der neuen Theorie die Lösung von drei recht unterschiedlichen Problemen: 
1. Systematik der Elementarteilchen, d. h., die neue Theorie soll uns ein Klassifikationsschema, vergleichbar dem Periodischen System der Elemente, liefern, das alle bekannten Elementarteilchen enthält und zugleich aussagt, ob es noch andere gibt und, wenn so, welche Eigenschaften sie haben;

2. Kopplungskonstanten, d. h. die neue Theorie soll die numerischen Werte der für die Wechselwirkung der verschiedenen Felder (und damit auch für die Lebensdauer der zugehörigen Teilchen) maßgebenden Kopplungskonstanten, die von der bisherigen Theorie prinzipiell nicht geliefert werden, zwangsläufig ergeben;

3. Massenspektrum, d. h. die neue Theorie soll die experimentell festgestellten Verhältniszahlen der Massen der verschiedenen Elementarteilchen liefern.

Es ist vollständig klar, daß von diesen drei Problemen allenfalls das erste in direkter Weise, d. h. durch Induktion und wissenschaftliche Abstraktion aus dem vorliegenden Erfahrungsmaterial und ohne wesentliche Zuhilfenahme der bestehenden Theorie und ihrer Widersprüche, gelōst werden kann. Aber ebenso wie das Periodische System der Elemente nach einer theoretischen Begründung verlangte, die erst durch die Quantenmechanik geliefert wurde, würde auch eine solche induktiv gewonnene Systematik der Elementarteilchen für die Entwicklung der Theorie noch recht wenig bedeuten.

Die neuen theoretischen Ansätze zielen denn auch ganz bewußt auf eine Verallgemeinerung der bisherigen Theorie ab. Ihre Diskussion von dem hier eingenommenen Standpunkt aus würde allerdings den Rahmen dieses Beitrages weit überschreiten. Ich möchte jedoch nicht meine Uberzeugung verschweigen, daß eine nicht-lokale Verallgemeinerung des bisherigen Feldbegriffes mehr in der allgemeinen Entwicklungsrichtung der Physik liegen dürfte als eine nicht-lineare Verallgemeinerung der Feldgleichungen. Vermutlich wird weder die eine noch die andere Verallgemeinerung alle genannten Probleme lösen, aber dies ist auch nicht zu erwarten, da diese Probleme sehr unterschiedlicher Natur sind und vielleicht nur in einer Folge von mehreren Verallgemeinerungen gelöst werden können. Insbesondere scheinen die neuen Erhaltungssätze der Elementarteilchenphysik, die für die Systematik der Elementarteilchen von grundlegender Bedeutung 
sind, in keinem unmittelbaren Zusammenhang mit dem vermutlich sehr viel tiefer liegendem Problem des Massenspektrums zu stehen.

Wie immer die Lösung dieser Probleme im einzelnen aussehen mag, es besteht kein Zweifel, daß sie vom mechanischen Materialismus noch weiter entfernt sein wird als die gegenwärtige Theorie und $\mathrm{da} B$ sie eine erneute Bestätigung und Weiterentwicklung des dialektischen Materialismus mit sich bringen wird. 